\title{
Fabrication Process of Large-area Morpho-color Flexible Film via Flexible Nano-imprint Mold
}

\author{
Akira Saito $^{1,2^{*}}$, Kosei Ishibashi ${ }^{1}$, Junpei Ohga ${ }^{1}$, \\ Yoshihiko Hirai ${ }^{3}$, and Yuji Kuwahara ${ }^{1,2}$ \\ ${ }^{1}$ Department of Precision Science \& Technology, Osaka University, \\ 2-1 Yamada-oka, Suita, Osaka 565-0871, Japan \\ ${ }^{2}$ RIKEN Harima Institute, 1-1-1, Kouto, Sayo-cho, Sayo-gun, Hyogo 679-5148, Japan \\ ${ }^{3}$ Department of Physics and Electronics Engineering, Osaka Prefecture University, \\ 1-1 Gakuen-cho, Naka-ku, Sakai, Osaka 599-8531, Japan \\ *saito@prec.eng.osaka-u.ac.jp
}

\begin{abstract}
Morpho butterfly's blue is the structural color given by optical interference, that contradicts its single coloration in too wide angular range. After proven the principle of the coloration by mimicking the specific nanostructures of their scales, we found the reproduced Morpho-color to serve wide applications, because it can produce a brilliant single color in wide angular range with high reflectivity for longtime without chemical pigment. We have then developed various techniques for practical applications of the specific color. One of the remaining issues is to mass-produce the large-area flexible film, which has long been difficult because of the process containing both nano-imprint and multilayer deposition. Thus, we developed a new process using the flexible mold and simple film-detachment system.
\end{abstract}

Keywords: Structural color, Morpho butterfly, Nano-imprint, Flexible mold, Film

\section{Introduction}

Morpho butterfly's metallic blue (Fig. 1 (a)) is a physical mystery that has both high reflectivity by interference and a single color in too wide angle $\left(> \pm 40^{\circ}\right.$ from the normal) without rainbow color. The mystery can be solved by a specific nanostructure on butterfly's scales. The optical principle is a blend of two opposed factors, the ordered and disordered structures [1]. As shown in Fig. 1 (b) left, their scale has tree-like complex 3D structure. Each tree has multilayer that is an ordered nanostructure, whereas the trees sit side by side with disordered height. The principles of this particular coloration is described as shown in Fig. 1 (c) corresponding to Fig. 1 (b). The principles are classified in five essences (Fig. 1 (c)). Although this explanation is common with our past reports $[1,2]$, it may help the reader's comprehension:

(1) The blue derives from multilayerinterference in a tree in vertical direction, i.e. alternating layers of high and low refractive index (protein: air =1.6: 1.0). (2) The blue is expanded in a wide angular range, because each tree's width is small $(\sim 300 \mathrm{~nm})$, which produces diffraction. (3) The trees sit side by side with disordered height. (Fig. 1 (b) left) and has disorder in-plane (Fig. 1 (b) right), as well. This disorder prevents a rainbow interference by interrupting the oblique optical path (Fig. 1 (c) left) and grating condition (Fig. 1 (c) right). (4) A narrow gap between the trees less than a wavelength of $\sim 450 \mathrm{~nm}$ (blue) generates high reflectivity. (5) The trees extend along the direction-Y in Fig. 1 (c) right, which makes a line-like anisotropy in top-view. This anisotropy gives a high reflectivity in a limited angle. Otherwise, if the structure has an isotropic randomness such as random dots in top view, the reflection will scatter isotropically, decreasing seriously the reflectivity at a viewing angle. 
(a)

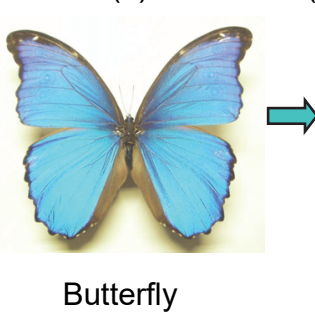

(b)

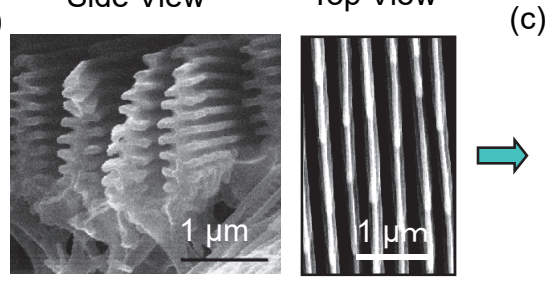

Nanoscale Observation (c)
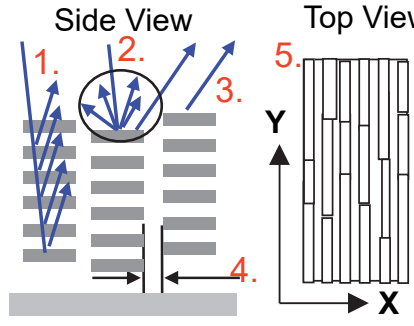

$\rightarrow$ Extract Optical Principle (d)

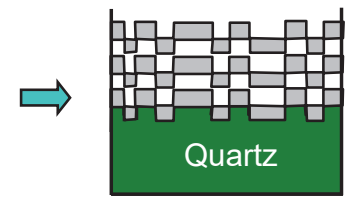

Nano-Fabrication

(e)

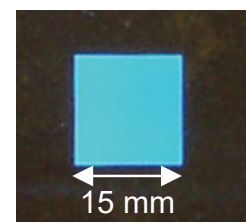

Fig. 1. Reproduction process (a-d) and replication process (f-h) of the Morpho-color. (a) Photo image of a typical Morpho butterfly (Morpho didius), (b) Cross-sectional (left) and top-view (right) SEM images of a single scale of Morpho didius, (c) Extracted model of the nanostructures corresponding to (b) where the numbers shown correspond to the text, (d) Schematic image of fabricated discrete multilayer after deposition of $\mathrm{TiO}_{2} / \mathrm{SiO}_{2}$ multilayer on the nano-pattern, (e) Visual aspect of the reproduced Morpho-blue. (f)(g) NIL process to replicate the nano-pattern shown in (d). (h) Mass-produced Morpho-structure after deposition of $\mathrm{TiO}_{2} / \mathrm{SiO}_{2}$ multilayer on the replicated nano-pattern.

These analytical principles needed an experimental proof. Although a single tree structure can be fabricated [3], it is difficult to achieve the trees in a wide area of a few $\mathrm{mm}$. Thus, we produced an optical material by extracting the principles (Fig. 1(c): multilayer, narrow width and gap of the trees, randomness, and anisotropy). We emulated the optical essence by dielectric multilayers $\left(\mathrm{TiO}_{2}\right.$ and $\left.\mathrm{SiO}_{2}\right)$ on an anisotropically nano-patterned quartz (Fig. 1 (d)). The structure was constructed by semiconductor techniques, which are electron beam (EB) lithography and reactive ion etching (RIE). Finally, we evidenced the analytical model by an engineering approach (Fig. 1 (e)). The details of the fabrication and estimations are shown elsewhere [2,4-6].

After proof of the principles (Figs. 1 (c)-(e)), the Morpho-color was found to serve wide applications. We have then developed various techniques for applications of this color for this decade: mass-production [7,8], control of angular width and color $[9,10]$, optical simulation on the nano-disorder [11,12], etc. [13]. For massproduction, nano-imprint lithography (NIL) is one of the most hopeful ways (Figs. 1 (f)-(h)).

One of the residual key subjects was to realize the large-area flexible film, because the past mass-productive Morpho-color materials by NIL were accompanied with thick substrate as shown in Figs. 1 (g)-(h), which has restricted the diversity of applications. Solid and flat substrate was needed because the stage in Fig. 1 (h) requires a substrate for homogeneous multilayer-deposition and heat endurance during deposition. Thus, the past NIL processes for Morpho-color material was restricted only to small area $\left(\sim 15 \times 15 \mathrm{~mm}^{2}\right)$, because the detachment of larger-area replica from the flat mold (Fig. $1(\mathrm{~g})$ ) needs large force that leads the mold into break or distortion. A unique duplication method for a large mold was then electro-forming for metallic nano-patterned mold [14]. On the other hand, we have recently developed a simple process to mass-fabricate a substrate-free Morpho-color material, such as color powders [15] and flexible film [16], although the size was still limited. Finally, we found that this new process serves to provide a large-area $\left(\sim 100 \times 100 \mathrm{~mm}^{2}\right)$ flexible film using a flexible mold. The process has to maintain the precision of the specific nano-pattern, and to be simple for applications. In this report, we describe a simple process to mass-produce a largearea flexible Morpho-color film using the flexible mold, especially for details on the process using polymers that were omitted in our previous report [17]. The fabricated film was found to have persistence against repeated strain, and to maintain the original optical properties of the Morpho-color. 


\section{Fabrication of flexible mold}

To complete large-area replication of the specific nano-pattern by NIL, a large-area flexible mold is key component. The flexible mold should be a precise replica of the original nano-pattern, robust, stable, and made of common material. One of the candidates is polydimethylsiloxane (PDMS) [18,19]. After replication of the nano-pattern on a large-area mold to a PDMS sheet, the PDMS- replica serves as a flexible mold in next step (Fig. 2). PDMS enables to avoid a retainable distortion of both mold and replica. Also we can detach gradually the PDMS replica under bent status from the original mold, by decreasing a detachment-area and detachment-force. Such gradual operation by flexibility gives good homogeneity to structure in wide area. PDMS replica has another advantage in throughput. Since the PDMS replica can be produced plurally, the second step of replication can be accomplished in parallel. Also the flexible mold can be applied to a cylindrical mold that is needed to roll-to-roll process, a typical highthroughput NIL system.

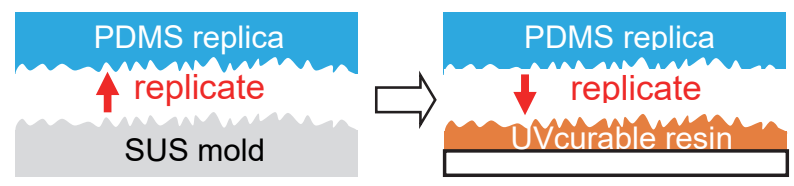

Fig. 2. Schematic image of the replication process to use the PDMS replica as a flexible mold.

First, a large-area SUS mold $\left(100 \times 100 \mathrm{~mm}^{2}\right.$, $0.5 \mathrm{~mm}$ in thick) having quasi-periodic nanopattern was fabricated by laser ablation [14]. For the PDMS precursor, Sylgard 184 (Dow Corning Toray Co., Ltd., Japan) was used [16], which is made by mixture of two materials, main and curing agents.

Before start the PDMS process, a treatment to ease the detachment of PDMS from the SUS mold was performed, i.e. cleaning and fluorine-polymer coating (Durasurf, Harves Co., Ltd., Japan) to reduce surface tension. PDMS precursor (mixture of two components) was poured with $2 \mathrm{~mm}$ in thick in a glass vessel, where the large-area SUS mold was put in advance. In a following heating process to harden the PDMS precursor into solid PDMS, higher temperature is better for shorter process. On the other hand, higher temperature gives larger adhesion force between PDMS and SUS mold. The heating temperature and time were then
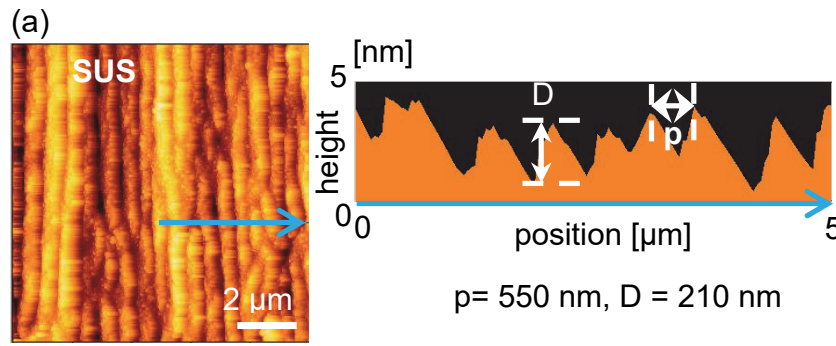

(b)
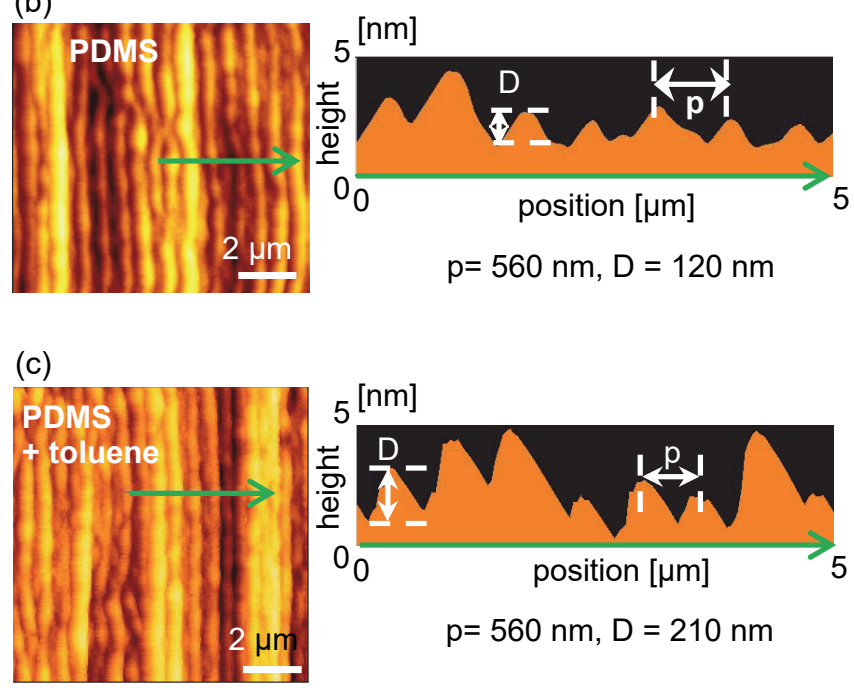

Fig. 3. AFM images (left) and cross-sectional profiles (right) along the arrow shown in AFM image for nano-patterns on (a) SUS mold, (b) initial PDMS replica in fail, (c) PDMS replica by use of toluene dilution. As a criteria of the replication quality, averaged pitch (p) and depth (D) are presented.

adjusted depending on the density of PDMS precursor.

After detachment of the solid PDMS replica from SUS mold, the quality of replication was estimated by atomic force microscopy (AFM) (SPI3800N, Hitachi High-Technologies Co., Ltd., Japan). Figures 3 (a) and (b) show the typical AFM image and cross-sectional profile along the arrow presented in the AFM image for SUS mold and PDMS replica, respectively. Quantitatively, an averaged value of pitch (p) and depth (D) of nano-patterns were evaluated, by picking up 20 measurement points.

In spite of our careful treatment, the first attempt to fabricate the PDMS replica was unsuccessful, where the replicated depth of nano-pattern was $120 \mathrm{~nm}$, which was much less than original value of $210 \mathrm{~nm}$ on the SUS mold. Since the pitch was not degraded on the PDMS replica, the unsuccessful phenomenon in the depth could be attributed to high viscosity of PDMS, which may limit PDMS to fill into the 
depth of nano-pattern (Fig. 4). Thus, we diluted the PDMS precursor by toluene [18] so that the toluene density becomes $60 \mathrm{wt} \%$.

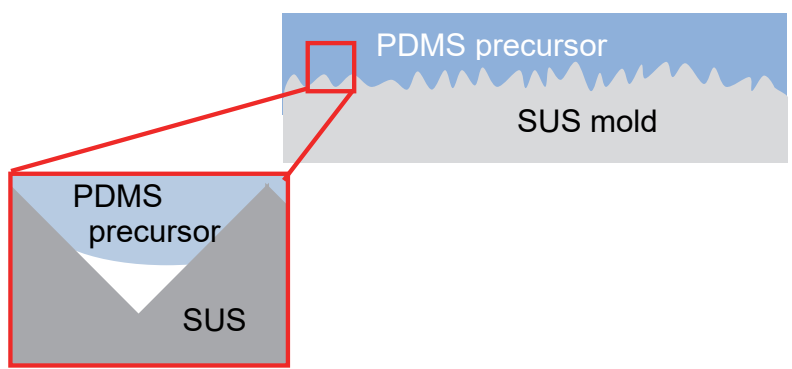

Fig. 4. Schematic image of the origin of fail in replication from SUS mold to PDMS precursor.

The diluted PDMS precursor was dipped on the SUS mold to cover fully the nano-patterned area. After evacuation to remove the toluene component in the PDMS precursor, PDMS was hardened by heating at $80{ }^{\circ} \mathrm{C}$ ( 1 hour), and final thickness of PDMS film was $\sim 0.5 \mathrm{~mm}$.

Since the PDMS replica made of diluted PDMS precursor was insufficient in strength due to its thinness, supporting PDMS was added using an original PDMS precursor without toluene. The supporting process is same as the first PDMS process without toluene, which was described above.

Finally, the nano-pattern on the SUS mold was replicated successfully on the PDMS replica, which was confirmed by AFM (Fig. 3 (c)). Both pitch (p) and depth (D) of the anisotropic groove pattern were maintained after replication.

\section{Fabrication of flexible film}

Using the fabricated PDMS replica as a mass-productive large-area flexible mold, replication of nano-pattern was attempted.

In our conventional NIL process $[7,8,16]$, the area of $15 \times 15 \mathrm{~mm}^{2}$ was imprinted from a nanopatterned quartz mold to the UV-curable resin put on a glass substrate. The quartz mold was treated by repeated cleaning and fluorine-polymer coating (Durasurf, Harves Co., Ltd., Japan) to reduce surface tension. In the present case, PDMS mold was not treated by fluorine-polymer, because the detachment of PDMS mold was clearly done without residual resin nor damage of resin on the glass. On the glass substrate (a commercial soda-lime glass of $140 \times 140 \mathrm{~mm}^{2}$ ), cleaning by ultrasonic in acetone and UV-ozone was performed to remove the contamination and make the resin to deposit on the glass as flat as possible.

In consideration of the radiation heat during the deposition process shown between Figs. 1(g) and 1(h), a heat-resistant UV-curable resin was used (PAK01, Toyo Gosei Co., Ltd.) [7,8]. The resin was dropped on the glass and PDMS mold was put on the resin.

For attachment of the PDMS mold on the resin on the glass substrate, the self-weight of the mold was simply enough in case of the small area of 15 $\times 15 \mathrm{~mm}^{2}$. In the present case, since the self-weight of the mold was not enough, a pressure was added manually and carefully to avoid the mixture of bubbles in the resin. Since the PDMS mold is flexible, the pressing performance by maintaining a homogeneous thickness of resin was achieved without problem, by bending the PDMS mold. For the UV treatment to harden the resin, there was no difference from our past reports [7,8].

After UV-NIL process, the quality of replicated pattern on the glass substrate was confirmed using AFM analysis (Fig. 5) in a same manner as shown in Fig. 3. Figure 5 shows that there is no difference in nano-patterns between the PDMS mold (Fig. 3 (c)) and the UV-curable resin.

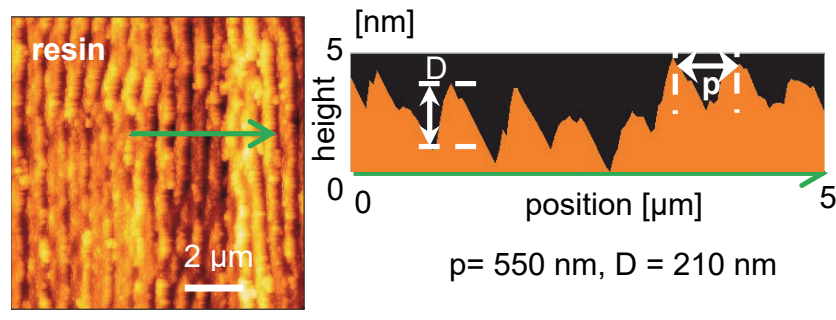

Fig. 5. AFM image (left) and cross-sectional profile (right) along the arrow shown in AFM image for nano-pattern replicated on the UV-curable resin.

The photo images of the fabricated PDMS mold and replica on UV-curable resin on the glass substrate are shown in Fig. 6.

(a)

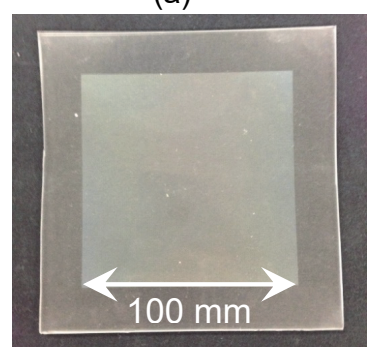

Fig. 6. Photo images of (a) PDMS replica (flexible mold) and (b) replica on the UV-curable resin. 
Next, Diamond-like Carbon (DLC) buffer layer was deposited on the replicated nano-pattern on the resin. This is to enhance the blue contrast by depositing an absorbing layer under the coloring multilayer [14]. On the buffer layer, the Morphocoloring film was made by multilayer-deposition on the nano-patterned resin $(\sim 10 \mu \mathrm{m}$ in thick) on the glass substrate. The parameter of multilayer was same as the past one composed of 14 layers of alternative $\mathrm{TiO}_{2}$ and $\mathrm{SiO}_{2}$ layers (Fig. 7 (a)) $[7,8]$. Figure 7 (b) shows the fabricated Morpho-color material on the replicated large-area nano-pattern on the solid glass substrate.

(a)

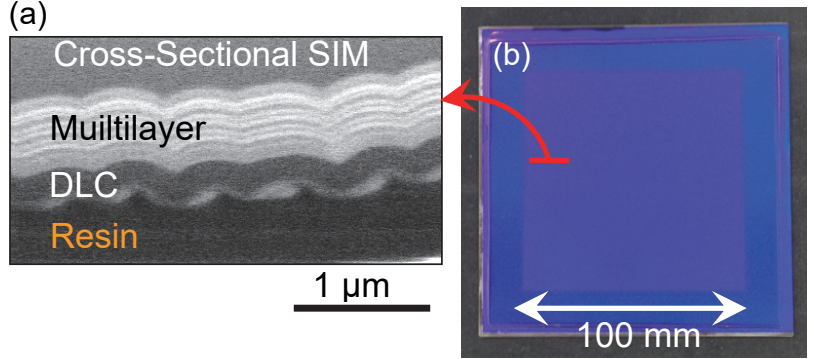

Fig. 7. (a) Cross-sectional SIM (Scanning Ion Microscope) image and (b) photo image of the reproduced Morpho-blue on a flat thick glass substrate.

Finally, the large-area flexible Morpho-color film was completed by detaching the coloring part composed of multilayer from the substrate. We have already achieved a simple process to detach the unbroken Morpho-color film from the substrate without additional process [16]. Though the size of the film was limited $\left(\sim 15 \times 15 \mathrm{~mm}^{2}\right)$ previously, the principle of detachment must be common, then an essentially same method as our previous report was used.

To protect the flexible thin film during detachment process, a cover was made by PDMS in advance ( $\sim 500 \mu \mathrm{m}$ in thick), because PDMS is common material having flexibility, strength and high transparency. To use a material commonly for the flexible mold and protection layer has an

(a)

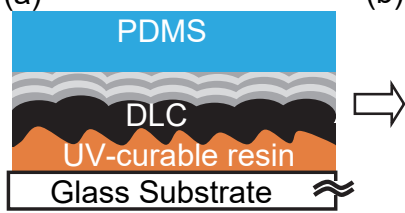

Fig. 8. Schematic image of the process to produce the flexible Morpho-color film coated by PDMS for protection. After immersion of the left part into the hot water, flexible film is detached from substrate. advantage for simple process. The PDMS coating was performed using Sylgard 184 with spin-coat, giving both heat-resistance and waterproof characteristics. Finally, the color film was detached from the substrate by immersion into the hot water $\left(80^{\circ} \mathrm{C}\right)$ (Fig. 8).

Figure 9 shows the large-area flexible Morphocolor film successfully obtained, which was not accompanied with break [15]. The fabricated large-area Morpho-color film was flexible and enduring against stress and strain.

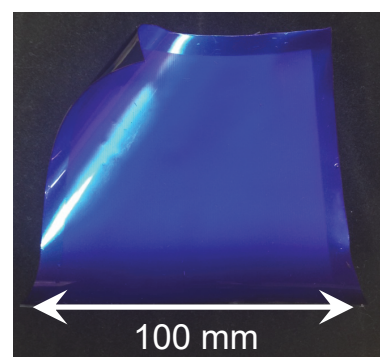

Fig. 9. Photo image of large-area flexible Morphocolor film.

\section{Optical properties of color-film}

The reflected blue coloration of the produced flexible film had a low angular dependence, maintaining the optical property of the original butterfly. To evaluate the optical properties quantitatively, reflective angular dependence was measured in a same manner as our past reports [15], i.e. a white incident light was introduced on a sample surface from normal direction. By scanning an optical fiber along the wide-reflective anisotropic direction (X-axis in Fig. 1 (c) right), the reflectivity on several wavelengths was measured using a spectrometer. Figures 10 (a), 10 (b), and 10 (c) show a comparison among the profiles from the large-area flexible Morpho-color film, conventional Morpho-color material on a solid plate, and the real Morpho butterfly's wing, respectively.

All profiles have wide angular range in blue, contrarily to the sharp specular peak for the usual multilayer [15] shown in Fig. 10 (d) that is an example of typical optical interference. Full width at half maximum (FWHM) for the wavelength of $460 \mathrm{~nm}$ are $48^{\circ}$ (Fig. 10 (a)), 42 (Fig. 10 (b)), and $66^{\circ}$ (Fig. 10 (c)), respectively. Smaller FWHM for the artificial color can be attributed to the quality of randomness in nanostructures [2,6,11], and importantly indicates that there is no degradation in the large-area 
(a) Large-area flexible Morpho-film

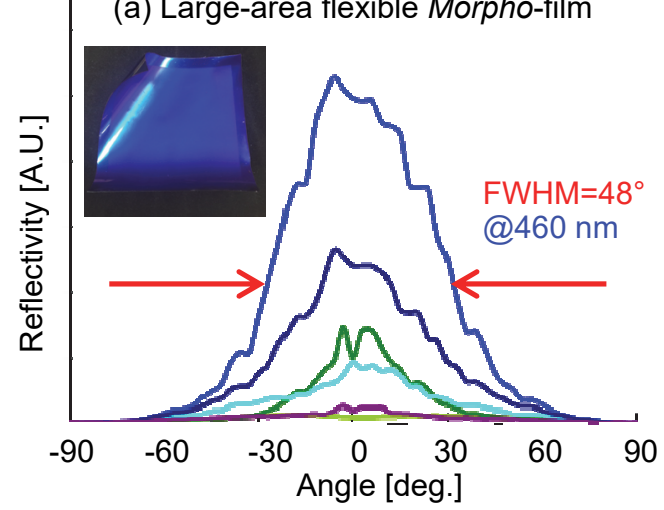

(c) Morpho Butterfly

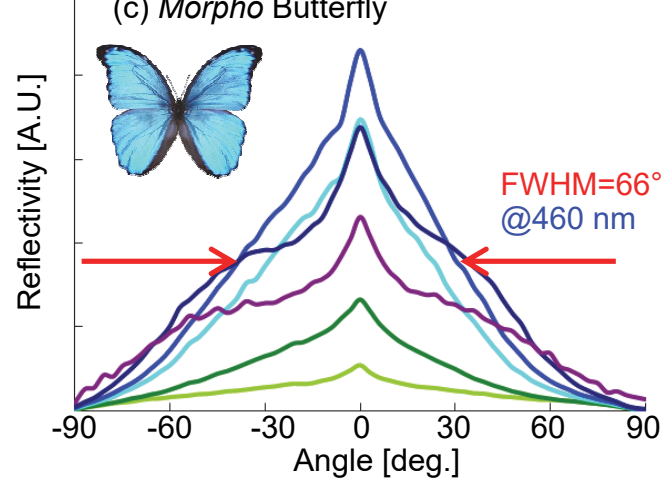

(b) Solid Morpho-plate

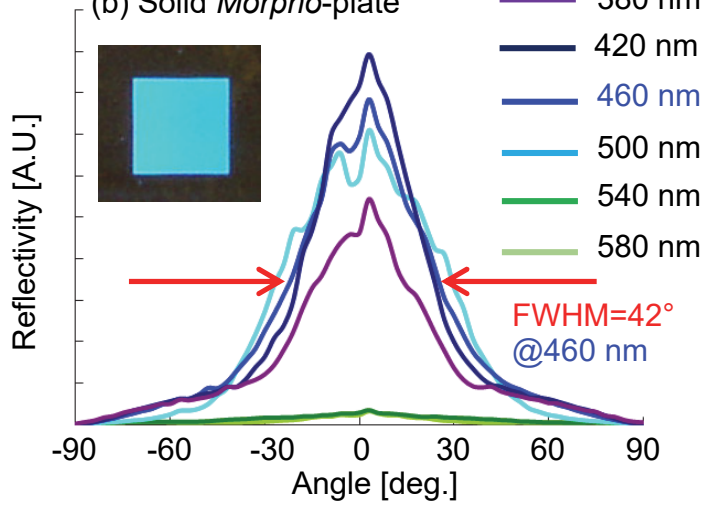

(d) Usual Multilayer

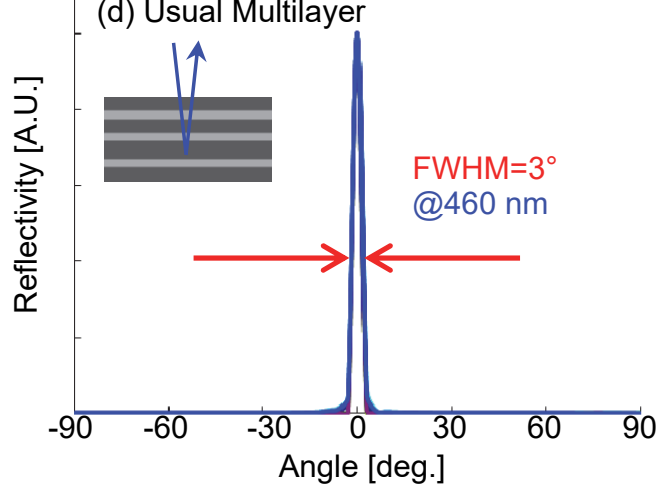

Fig. 10. Reflective angular dependence at various wavelengths for (a) large-area flexible Morpho-color film, (b) conventional Morpho-color material on a solid flat glass substrate, (c) Morpho Butterfly, and (d) usual multilayer.

flexible film from the conventional solid Morpho-color material. Figure 10 (a) shows no specular sharp peak, indicating that the flexible Morpho-color film is not affected by a mirror-reflection from PDMS coating. The result with no serious degradation of reflectivity means no serious damages in the color part (multilayer) even after detachment process and bending. The optical property was persistent against repeated bending of more than 100 times.

\section{Conclusions}

The Morpho-color enables both wide reflective angle and high reflectivity of single color that are usually not compatible. These characteristics are produced by the discrete dielectric multilayer containing specific nano-disorder. By aiming at the industrial applications of this specific color, large-area flexible Morpho-color film was produced by developing a NIL-based massproduction process, especially by creating a flexible mold.

Since the Morpho-color is based on a specific random and anisotropic nano-pattern, the massproduction process needs NIL method. The coloring multilayer was homogeneously deposited on a nano-imprinted resin attached on a flat thick substrate. The size to replicate the nano-pattern by NIL on a flat thick substrate was restrained to $\sim 15 \times 15 \mathrm{~mm}^{2}$ due to the large force to detach the replica from the mold. The flexible mold enabled to release the limitation of size by decreasing the detachment-force. Finally, using our recent post-NIL process for substrate-free material $[15,16]$, a large-area $\left(\sim 100 \times 100 \mathrm{~mm}^{2}\right)$ flexible Morpho-color film was realized by simple process. The optical property of the product was found to maintain basically the original property after detachment from the substrate and PDMS coating, i.e. brilliant blue with high reflectivity, a wide angular range of single color, anisotropy of the brilliance, and a smooth hue without fringe-like sharp specular reflection $[2,6]$.

This coloration has wide potential applications: it produces color without pigment, makes conspicuous hue qualitatively impossible by pigment. It has advantages in fading-free, and can produce any color by changing parameters of the multilayer using only two different materials. Ecological merits are pigment-free and saving energy (high reflectivity) that are fit to the use of 
posters or displays. Other various applications are relevant to this color: cosmetics, decorations, textures, paints, security, etc.

Morpho-color is a typical example in a field of "biomimetics", and then will be expected to have much importance in near future for sustainable world [20]. The simple processes reported here will serve to develop generally thin film, not only for Morpho-color materials.

\section{Acknowledgement}

This study was supported by KAKENHI (Grand-in-Aid from the Ministry of Education, Sports, Culture, Science and Technology) No. 26289249 and No. 21360033.

\section{References}

1. S. Kinoshita, S. Yoshioka, and K. Kawagoe, Proc. R. Soc. Lond. B, 269 (2002) 1417.

2. A. Saito, S. Yoshioka, and S. Kinoshita, Proc. SPIE, 5526 (2004) 188.

3. K. Watanabe, T. Hoshino, K. Kanda, Y. Haruyama, and S. Matsui, Jpn. J. Appl. Phys., 44 (2005) L48.

4. A. Saito and S. Kinoshita, Japan Patent JP 2001-241496 (2001).

5. A. Saito and S. Yoshioka, Japan Patent JP 2003-391572 (2003).

6. A. Saito, in "Structural Colors in Biological Systems", S. Kinoshita \& S. Yoshioka, Eds., Osaka University Press, Osaka (2005) p.287295.

7. A. Saito, Y. Miyamura, M. Nakajima, Y. Ishikawa, K. Sogo, Y. Kuwahara, and Y. Hirai, J. Vac. Sci. Technol. B, 24 (2006) 3248.

8. A. Saito, Y. Miyamura, Y. Ishikawa, J. Murase, M. Akai-Kasaya, and Y. Kuwahara, Proc. SPIE,
7205 (2009) 720506.

9. A. Saito, Y. Ishikawa, Y. Miyamura, M. Akai-Kasaya, and Y. Kuwahara, Proc. SPIE, 6767 (2007) 676706.

10. A. Saito, in "Biomimetic Photonics", O. Karthaus, Ed., Taylor \& Francis Publisher (2012) p.96-115.

11. A. Saito, M. Yonezawa, J. Murase, S. Juodkazis, V. Mizeikis, M. Akai-Kasaya, and Y. Kuwahara, J. Nanosci. Nanotechnol., 11 (2011) 2785.

12. A. Saito, in "Biomimetic Photonics", O. Karthaus, Ed., Taylor \& Francis Publisher (2012) p.226-242.

13. A. Saito, Sci. Technol. Adv. Mater., 12 (2011) 64709.

14. A. Saito, J. Murase, M. Yonezawa, H. Watanabe, T. Shibuya, M. Sasaki, T. Ninomiya, S. Noguch, M. Akai-Kasaya, and Y. Kuwahara, Proc. SPIE, 8339 (2012) 83390C.

15. A. Saito, K. Ishibashi, M. Akai-Kasaya, and Y. Kuwahara, Proc. SPIE, 9429 (2015) 942912.

16. A. Saito, K. Ishibashi, J. Ohga, and Y. Kuwahara, Proc. SPIE, 10162 (2017) 101620U.

17. A. Saito, K. Ishibashi, J. Ohga, Y. Hirai, and Y. Kuwahara, Proc. SPIE, 10593 (2018) 105930C.

18. N. Koo, M. Bender, U. Plachetka, A. Fuchs, T. Wahlbrink, J. Bolten, and H. Kurz, Microelectron. Eng., 84 (2007) 904.

19. S Aura, V Jokinen, L Sainiemi, M Baumann, and S Franssila, J. Nanosci. Nanotechnol., 9 (2009) 6710.

20. A. Lakhtakia and R. Jose Martin-Palma, Eds., "Engineered Biomimicry", Elsevier (2013). 